\title{
NONOSCILLATION THEOREMS FOR A CLASS OF NONLINEAR DIFFERENTIAL EQUATIONS( $\left.{ }^{1}\right)$
}

\author{
BY \\ RICHARD A. MOORE AND ZEEV NEHARI
}

1. The differential equations to be considered in this paper are of the form (1)

$$
v^{\prime \prime}+p(x) y^{2 n+1}=0,
$$

where $p(x)$ is positive and continuous in $(0, \infty)$ and $n$ is a positive integer.

While considerable insight into the nature of the solutions of (1) can be gained by direct manipulation of the equation, it will be seen that a more penetrating discussion requires the study of an appropriate variational problem whose solutions must satisfy (1). The obvious choice, namely, the problem

$$
\delta \int_{a}^{b}\left[(n+1) y^{\prime 2}-p y^{2 n+2}\right] d x=0
$$

of which (1) is the Euler equation, proves to be utterly unsuitable for our purposes. The main reason for this is the fact that the family of extremals of this problem which pass through a point do not form a field and that, therefore, the classical sufficient criteria for the existence of extrema become unapplicable. We shall show that the proper problem is that of minimizing the generalized Rayleigh quotient

$$
J(y)=\left(\int_{a}^{b}{y^{\prime}}^{2} d x\right)^{n+1} / \int_{a}^{b} p y^{2 n+2} d x
$$

for suitable homogeneous boundary conditions. It will be found that this problem has a solution and that this solution, if properly normalized, must satisfy (1). This connection between equation (1) and the functional (3) thus provides an additional tool for the investigation of the solutions of (1). Besides, it is a source of integral inequalities in those cases in which the general solution of (1) can be obtained explicitly. A few examples of this type will be exhibited in $\$ 10$.

2. We begin with a result of an essentially geometric nature which may be regarded as a substitute for the separation property of the solutions of a linear equation.

Received by the editors August 4, 1958. search.

(1) This research was supported by the United States Air Force Office of Scientific Re- 
Lemma I. Let $F(x, y)$ be continuous for $0<x<x$ and $-\infty<y<\infty$, and let $u(x), v(x), w(x)$ be solutions of the differential equation

$$
y^{\prime \prime}+F(x, y)=0 .
$$

If, in an interval $[a, b](0<a<b<\infty), u(x) \leqq v(x) \leqq w(x)$ and if $F(x, y)$ is a convex function of $y$ for min $u<y<\max w$ and any fixed positive $x$, then

$$
\phi(x) \equiv(w-v)\left(v^{\prime}-u^{\prime}\right)-(v-u)\left(w^{\prime}-v^{\prime}\right)
$$

is a strictly increasing function in $[a, b]$ except when equation (4) is linear.

Proof. It is easily confirmed that

$$
\phi^{\prime}(x)=w v^{\prime \prime}-v w^{\prime \prime}+u w^{\prime \prime}-w u^{\prime \prime}+v u^{\prime \prime}-u v^{\prime \prime} .
$$

Hence, if we abbreviate $F(x, y)$ to $F(y)$ and use (4), we obtain after some rearrangement

$$
\phi\left(x_{2}\right)-\phi\left(x_{1}\right)=\int_{x_{1}}^{x_{2}}\{(v-u)[F(w)-F(v)]-(w-v)[F(v)-F(u)]\} d x,
$$

where $a \leqq x_{1}<x_{2} \leqq b$. Since $u<v<w$ and $F(y)$ is convex, we have

$$
\frac{F(v)-F(u)}{v-u} \leqq \frac{F(w)-F(u)}{w-u},
$$

with equality only if the points $(u, F(u)),(v, F(v)),(w, F(w))$ lie on a straight line. It follows that the integrand is positive unless $F(x, y)$ is linear in $y$ in the range concerned. This completes the proof.

We remark that Lemma 1 applies to equation (1) only if the solutions $u, v, w$ are non-negative in $[a, b]$.

The following result is an immediate consequence of Lemma I.

Lemma II. Let $F(y, x)$ satisfy the hypotheses of Lemma I. If $u(x, v(x), w(x))$ are solutions of (4) such that $u(x)<w(x)$ and $v(x)<w(x)$ in $[a, b]$, then the curves $\zeta=u(x)$ and $\zeta=v(x)$ cannot intersect in $[a, b]$ more than once.

Indeed, if there are more points of intersection there will exist two points $x=x_{1}$ and $x=x_{2}\left(a \leqq x_{1}<x_{2} \leqq b\right)$ such that $u\left(x_{1}\right)=v\left(x_{1}\right), u\left(x_{2}\right)=v\left(x_{2}\right)$ and, say, $v(x)>u(x)$ in $\left(x_{1}, x_{2}\right)$. If $\phi(x)$ is the expression defined in (5), we then have

$$
\phi\left(x_{1}\right)=\left[w\left(x_{1}\right)-v\left(x_{1}\right)\right]\left[v^{\prime}\left(x_{1}\right)-u^{\prime}\left(x_{1}\right)\right]>0
$$

and

$$
\phi\left(x_{2}\right)=\left[w\left(x_{2}\right)-v\left(x_{2}\right)\right]\left[v^{\prime}\left(x_{2}\right)-u^{\prime}\left(x_{2}\right)\right]<0 .
$$

This contradicts Lemma I, and thus proves Lemma II.

If $y(x)$ is a solution of (1) for which $y(a)=y(b)=0$ and $y(x)>0$ in $(a, b)$, we may conclude from Lemma II (and the fact that $y \equiv 0$ is a trivial solution 
of (1)) that any solution curve $\zeta=u(x)$ of (1) which satisfies $u(a)=0,0<u^{\prime}(a)$ $<y^{\prime}(a)$ must intersect the curve $\zeta=y(x)$ in $(a, b)$. Moreover, if $v(x)$ is another solution of (1) and $v(a)=0,0<v^{\prime}(a)<u^{\prime}(a)<y^{\prime}(a)$, the curves $\zeta=u(x)$ and $\zeta=v(x)$ cannot intersect in the interval bounded by $x=a$ and the first point of intersection of $\zeta=y(x)$ with either of these curves. If this is combined with a continuity argument and some additional elementary considerations, it is not difficult to show that for any $a, b$ such that $0<a<b<\infty$ equation (1) has a solution which vanishes for $x=a$ and $x=b$ and is positive in $(a, b)$. We omit this argument, since the existence of such solutions will be established by the solution of the variational problem of $\S 6$.

If it were true that this solution is uniquely determined by its two consecutive zeros, we could conclude that the first zero in $(a, \infty)$ of a solution $y(x)$ of (1) which vanishes at $z=a$ will move to the right if $\left|y^{\prime}(a)\right|$ decreases, and this would lead to a very considerable simplification of the theory of equation (1). While all the "reasonable" examples of such equations for which we can obtain the general solution show this regular behavior, the following example shows that there may be exceptions. Let $y_{0}(x)$ be the solution of

$$
y^{\prime \prime}+y^{2 n+1}=0
$$

which satisfies $y(0)=0, y^{\prime}(1)=0$, and $y^{\prime}(x)>0$ for $0 \leqq x<1$. We note that $y_{0}(2-x)$ is also a solution of (6) and, moreover, is identical to $y_{0}(x)$; thus $y_{0}^{\prime}(x)<0$ for $1<x \leqq 2$. Now for any number $c, c y_{0}\left(c^{n} x\right)=y(x, c)$ is a solution of (6), and

$$
\begin{aligned}
& \frac{\partial y}{\partial c}(1, c)=y_{0}\left(c^{n}\right)+n c^{n} y_{0}^{\prime}\left(c^{n}\right), \\
& \frac{\partial y}{\partial x}(1, c)=c^{n+1} y_{0}^{\prime}\left(c^{n}\right),
\end{aligned}
$$

are continuous functions of $c$. By our above remarks and these formulas $(\partial y / \partial x)(1, c)$ changes sign at $c=1$, and $(\partial y / \partial c)(1,1)>0$; therefore, by a continuity argument there exist two numbers $c_{1}$ and $c_{2}, 0<c_{1}<1<c_{2}$, such that

$$
\begin{array}{ll}
0<y\left(x, c_{1}\right)<y_{0}(x)<y\left(x, c_{2}\right), & 0<x \leqq 1, \\
0 & <\frac{\partial y}{\partial x}\left(1, c_{1}\right)=-\frac{\partial y}{\partial x}\left(1, c_{2}\right) .
\end{array}
$$

We set $p(x) \equiv 1$ in $[0,1]$ and $[\alpha, a+1]$, where

$$
\alpha=1+\frac{y\left(1, c_{2}\right)-y\left(1, c_{1}\right)}{\frac{\partial y}{\partial x}\left(1, c_{1}\right)},
$$

and $p(x) \equiv 0$ in $(1, \alpha)$. Although this function $p(x)$ is neither positive nor continuous in the interval considered, it is not difficult to show that the small 
change of $p(x)$ required to remove these deficiencies will not affect the basic behavior of the solution. The two solutions of equation (1) $y_{1}(x)$ and $y_{2}(x)$ are defined as follows

$$
\begin{aligned}
y_{1}(x) & =y_{0}(x), & 0 \leqq x \leqq 1 \\
& =y_{0}(1), & 1 \leqq x \leqq \alpha \\
& =-y_{0}(x-\alpha-1), & \alpha \leqq x \leqq \alpha+1 . \\
y_{2}(x) & =y\left(x, c_{1}\right), & 0 \leqq x \leqq 1 \\
& =y\left(1, c_{1}\right)+\frac{\partial y}{\partial x}\left(1, c_{1}\right)(x-1), & 1 \leqq x \leqq \alpha \\
& =-y\left(x-\alpha-1, c_{2}\right), & \alpha \leqq x \leqq \alpha+1 .
\end{aligned}
$$

Both $y_{1}(x)$ and $y_{2}(x)$ are continuous and have continuous derivatives on $[0, \alpha+1]$ which is all that is required of solutions of equation (1) for piecewise continuous $p(x)$. It may be remarked that there is still a third solution, positive on $(0, \alpha+1)$ and zero at the end points, namely, $y(\alpha+1-x)$.

3. We now turn to the discussion of the oscillatory behavior of the solutions of (1). Here, certain precautions are necessary. It will be shown in $\S 8$ that, for any given $a, b(0<a<b<\infty)$, there always exist solutions of (1) which have an arbitrarily given finite number of zeros in $(a, b)$. Even if there is no solution of (1) with an infinite number of zeros in $(a, \infty)$, it is therefore not possible to find a value $x=x_{0}$ such that the number of zeros of all solutions in $\left(x_{0}, \infty\right)$ is bounded. Another complication is the fact that some solutions may have an infinite number of zeros in $(a, \infty)$, while other solutions of the same equation do not oscillate at all in the same interval. This behavior may be illustrated by the example of the equation

$$
y^{\prime \prime}+\frac{y^{2 n+1}}{4 x^{n+2}}=0
$$

which is also instructive in other respects. The general solution of (7) is

$$
y=(x)^{1 / 2} u(\log x),
$$

where $u(t)$ is the general solution of

$$
4 \ddot{u}+u^{2 n+1}-u=0,
$$

which can be found expicitly. All solutions of (9) are solutions of

$$
4 \dot{u}^{2}+\frac{u^{2 n+2}}{n+1}-u^{2}=4 b^{2}+\frac{a^{2 n+2}}{n+1}-a^{2}=A
$$

where $u(0)=a, \dot{u}(0)=b$. If $A=0$ and, say, $b<0$ and $a>0$, then the solution is given by 


$$
t=2 \int_{u}^{a} \frac{d s}{s\left(1-\frac{s^{2 n}}{n+1}\right)^{1 / 2}} .
$$

$u(t)$ is initially decreasing and by (11) will either decrease monotonically to zero as $t \rightarrow \infty$ or $u^{\prime}\left(t_{0}\right)=0$ for some finite value $t_{0}$. In this last case $u\left(t_{0}\right)$ $=(n+1)^{1 / 2 n}$, but $A=0$ also implies that $(n+1)^{1 / 2 n}>a>u\left(t_{0}\right)$, which is impossible.

The function $u(-t)=w(t)$ is also a solution of (9) and is given by

$$
\iota=2 \int_{a}^{w} \frac{d s}{s\left(1-\frac{s^{2 n}}{n+1}\right)^{1 / 2}},
$$

from which it is evident that $w$ increases monotonically and assumes the value $(n+1)^{1 / 2 n}$ for some finite value $t_{1}$. Since $w^{\prime}\left(t_{1}\right)=0$, the function $w\left(2 t_{1}-t\right)$, being a solution of $(9)$, is identical to $w(t)$; thus $w(t)$ decreases monotonically to zero as $t$ runs from $t_{1}$ to $+\infty$. Thus the curve $\zeta=u(t)$ is found to be symmetric about $t=-t_{1}$ and $u(t)$ to take all values $a$ such that $0<a \leqq(n+1)^{1 / 2 n}$. This last remark shows that all solutions in the case $A=0$ are given by $\pm u(t+\tau)$ for any $\tau$.

The asymptotic behavior of $u(t)$ near $t=\infty$ is expressed by

$$
\begin{aligned}
\lim _{t \rightarrow \infty}\left[\frac{t-t_{1}}{2}-\log \frac{(n+1)^{1 / 2 n}}{u(t)}\right] & =\int_{0}^{(n+1)^{1 / 2 n}}\left\{\left[1-\frac{s^{2 n}}{n+1}\right]^{-1}-1\right\} \frac{d s}{s} \\
& =\gamma>0 ;
\end{aligned}
$$

i.e., $u(t) \sim a_{0} \exp \left\{-\left(t-t_{1}\right) / 2+\gamma\right\} \quad\left(a_{0}=(n+1)^{1 / 2 n}\right)$. As (8) shows, the corresponding solution $y(x)$ of (7) has the initial data

$$
y(1)=a, \quad y^{\prime}(1)=\frac{1}{2} a+b>0 .
$$

Evidently, there are two positive bounded solutions through every point $(1, a), 0<a<a_{0}$, asymptotic to $a_{0} e^{+t_{1} / 2}$ and $a_{0} e-t_{1 / 2}$. For the point $\left(1, a_{0}\right)$ there is exactly one bounded solution, asymptotic to $a_{0} e^{\gamma}$.

All other solutions of (9) are obtained when $A \neq 0$. A discussion similar to the above shows that all these solutions are periodic. If $A>0, u(t)$ has an infinite number of zeros in $(0, \infty)$. However, if $A<0, \zeta=u(t)$ oscillates infinitely often without crossing the horizontal axis. Since between a maximum and a minimum of $\zeta=u(t)$ there must be a point such that $\ddot{u}=0$, (9) shows that $\zeta=u(t)$ intersects the solution $u \equiv 1$ infinitely often. We thus obtain the following picture for the totality of solutions of (7) which pass through the point $(1, a)$. If $y^{\prime}(1)=a / 2 \pm b$, where $b$ is the positive solution of $(10), y(x)$ is 
positive and tends to a finite limit as $x \rightarrow \infty$. For $a / 2-b<y^{\prime}(1)<a / 2+b$, $y^{\prime}(1) \neq 1 / 2$, the solutions are positive and intersect the solution $x^{1 / 2}$ an infinity of times. For $y^{\prime}(1)<a / 2-b$ and $y^{\prime}(1)>a / 2+b$, the solutions are oscillatory.

4. This example raises the question as to the proper definition of a "nonoscillatory equation." Should one merely require the existence of at least one nonoscillatory solution, or should this term be reserved for an equation none of whose solutions have an infinity of zeros? While we cannot give a completely satisfactory answer to this question, the following remarks may throw some light on the matter. It will be observed that none of the nonoscillatory solutions of (7) vanish even once in the entire interval of continuity $(0, \infty)$ of the coefficient of the equation. As will be seen later, the existence of a nonoscillatory solution which vanishes at least once in the interval of continuity of the coefficient is a considerably stronger condition than the mere existence of a solution which does not oscillate. Indeed, a good case can be made for regarding as properly nonoscillatory only the solutions of the former type. Whether an equation (1) may have both oscillatory solutions and nonoscillatory of this restricted type is a question we cannot answer.

That the mere existence of nonoscillatory solutions-regardless of typegives but little indication of the behavior of the totality of the solutions of (1) is shown by the following recent result of Atkinson [1], which gives a complete and elementary answer to this question of existence.

Theorem I (AtKInson). Equation (1) has a nonoscillatory solution if, and only if,

$$
\int_{a}^{\infty} x p(x) d x<\infty
$$

Indeed, even more is true. It was shown in [1] that (12) insures the existence of a solution $y(x)$ such that $y(x) \rightarrow 1$ for $x \rightarrow \infty$. Since, for any constant $\alpha$, the function $\alpha y(x)$ likewise satisfies an equation of type (1), it follows that (1) has solutions $y(x)$ such that $y(x) \rightarrow \beta$ for $x \rightarrow \infty$, where $\beta$ is an arbitrary constant. Hence, (12) guarantees even an infinity of nonoscillatory solutions. Finally, it follows from the necessary and sufficient character of (12) that if (1) has one nonoscillatory solution it must have an infinite number of them.

If it is known that (1) has a nonoscillatory solution of sufficiently fast growth, (12) can be strengthened. A modified form of the argument showing the necessity of (12) yields the following result.

THEOREM II. If (1) has a nonoscillatory solution for which

$$
\int^{\infty} \frac{x^{\nu} d x}{[y(x)]^{2 n}}<\infty, \quad \nu>-1
$$


then

$$
\int^{\infty} x^{\nu+2} p(x) d x<\infty .
$$

If $y(x)$ is a solution of (1) such that $y(x)>0$ for $a \leqq x<\infty$, it follows from the fact that $y^{\prime \prime}(x)<0$ that $y^{\prime}(x)>0$ in $[a, \infty]$. Equation (1) may be brought into the form

$$
\frac{y^{\prime}(a)}{y^{2 n+1}(a)}=\frac{y^{\prime}(x)}{y^{2 n+1}(x)}+(2 n+1) \int_{a}^{x} \frac{y^{\prime}}{y^{2 n+2}} d t+\int_{a}^{x} p d t, \quad a<x,
$$

which shows that

$$
\frac{y^{\prime}(a)}{y^{2 n+1}(a)} \geqq \int_{a}^{x} p d l .
$$

Integrating over $a$ from $a$ to $x$, we obtain

$$
\frac{1}{y^{2 n}(a)} \geqq \frac{1}{y^{2 n}(x)}+2 n \int_{a}^{x}(t-a) p(t) d \iota \geqq 2 n \int_{a}^{x}(t-a) p(t) d t .
$$

Since $x$ may be taken arbitrarily large, this is equivalent to (12). Multiplying the inequality

$$
\frac{1}{y^{2 n}(s)} \geqq 2 n \int_{0}^{x}(t-s) p(t) d t
$$

by $(s-a)^{v}$ and integrating over $s$ from $a$ to $x$, we have

$$
\int_{a}^{x} \frac{(s-a)^{\nu}}{y^{2 n}(s)} d s \geqq \frac{2 n}{(\nu+1)(\nu+2)} \int_{a}^{x}(s-a)^{\nu+2} p(s) d s .
$$

Since $x$ may be taken arbitrarily large, this proves Theorem II.

5. It was shown in Atkinson [1] that no solution of (1) will be oscillatory if

$$
\int^{\infty} x^{2 n+1} p(x) d x<\infty,
$$

provided $p(x)$ is a differentiable and nonincreasing function of $x$. Again, this condition implies much more than mere nonoscillation. As the following result shows, (14) is-without any monotonicity or differentiability conditions - equivalent to the existence of a certain simple type of solution.

THEOREM III. Equation (1) has solutions for which

$$
\lim _{x \rightarrow \infty} \frac{y(x)}{x}=\alpha>0
$$

if, and only if, condition (14) is satisfied. 
The necessity of condition (14) is obvious, since

$$
y^{\prime}(a)=y^{\prime}(b)+\int_{a}^{b} p y^{2 n+1} d x
$$

and $y^{\prime}(b)>0$ if $y(x)>0$ in $(a, \infty)$.

To show sufficiency, we assume that (14) holds and choose a value of $a$ which is large enough so that

$$
\int_{a}^{\infty} x^{2 n+1} p(x) d x<\epsilon
$$

where the positive constant $\epsilon$ may be taken arbitrarily small. We define a solution $y(x)$ of $(1)$ by the initial conditions $y(a)=0, y^{\prime}(a)=c$, where $0<c \leqq 1$, and we assert that $y(x)$ will be positive in $(a, \infty)$ and will show the asymptotic behavior (15).

If $y(x)$ has a zero in $(a, \infty)$, there must exist a value $x=b$ in $(a, \infty)$ such that $y(b)=0$ and $y(x)>0$ in $(a, b)$. Since $y(x)$ is necessarily concave in the latter interval, we have there $y(x) \leqq y^{\prime}(a)(x-a)$ and we may conclude from (16) that

$$
y^{\prime}(a) \leqq y^{\prime}(x)+\left[y^{\prime}(a)\right]^{2 n+1} \int_{a}^{x}(x-a)^{2 n+1} p(x) d x, \quad a<x<b .
$$

In view of (17) and the assumption $0<y^{\prime}(a)<1$, it follows that

$$
y^{\prime}(a) \leqq y^{\prime}(x)+\epsilon y^{\prime}(a),
$$

i.e.,

$$
(1-\epsilon) y^{\prime}(a) \leqq y^{\prime}(x) .
$$

This proves that $y^{\prime}(x)>0$ in the entire interval $(a, b)$, provided $\epsilon<1$ and thus shows that $y(x)$ cannot vanish for $x=b$. Since, for positive $y(x), y^{\prime}(x)$ decreases monotonically we see, moreover, that $\lim y^{\prime}(x)$ for $x \rightarrow \infty$ exists and is positive. This completes the proof of Theorem III.

It will be noted that the nonoscillatory solution whose existence was proved in the preceding argument does have a zero at $x=a$. This shows that condition (14) is strong enough to produce the type of proper nonoscillatory solution discussed in $\S 4$. There is yet another aspect of condition (14) which is worthy of note. According to Lemma II, it cannot happen that there are two solutions $u(x)$ and $v(x)$ of (1) such that $v(a)=v(b)=0(0<a<b<\infty)$ and $0<v(x)<u(x)$ in $(a, b)$. In many cases, this restriction will remain in force even if $b=\infty$, i.e. if $u(x)$ and $v(x)$ are nonoscillatory in $(a, \infty)$. It is, for instance, easily confirmed that this is true if $u(x)$ remains bounded as $x \rightarrow \infty$. However, if condition (14) holds, there will exist pairs of solutions $u(x), v(x)$ such that $v(a)=0$ and $0<v(x)<u(x)$ in $(a, \infty)$. 
We assume, for simplicity, $u(a)=0$; the case in which $u(a)>0$ can be handled by a trivial modification of the same argument. We take $0<v^{\prime}(a)$ $<u^{\prime}(a)<1$, where $a$ is taken sufficiently large so that (17) is satisfied. By (18), we have

$$
u^{\prime}(x)-v^{\prime}(x)>u^{\prime}(a)(1-\epsilon)-v^{\prime}(a) .
$$

Hence, if $v^{\prime}(a)$ is taken small enough, the left-hand side of this inequality is positive for all $x>a$, and we may conclude that $u(x)>v(x)$ in $(a, \infty)$.

According to Theorem III, equation (1) has solutions with the asymptotic behavior (15) if condition (14) holds. As shown in [1], this equation also has bounded solutions. The following theorem which generalizes a result of Hille for the case $n=0$ shows that no other types of nonoscillatory solutions are possible.

THEOREM IV. If condition (14) holds, all nonoscillatory solutions of (1) are either bounded, or else have the asymptotic behavior (15).

Proof. Let $y(x)$ be a solution of (1) for which $y(x)>0$ for $a \leqq x<\infty$. By (14), it is possible to choose a value $B$ such that for all $b \geqq B$,

$$
\left[2 y^{\prime}(a)\right]^{2 n} \int_{b}^{\infty} p(x) x^{2 n+1} d x<\epsilon,
$$

where $\epsilon$ is an arbitrarily small positive constant. We replace (1) by the equivalent integral equation

$$
y(x)=y(b)+\int_{b}^{x}(t-b) p(t) y^{2 n+1}(t) d t+(x-b) y^{\prime}(x)
$$

and we note that $y(t) \leqq y(x)$ for $b \leqq t \leqq x$. Hence,

$$
1 \leqq \frac{y(b)}{y(x)}+\int_{b}^{x} t p(t) y^{2 n}(t) d t+\frac{x y^{\prime}(x)}{y(x)} .
$$

If $B$ is also taken sufficiently large so that $B y^{\prime}(a) \geqq y(a)$, we have

$$
y(t) \leqq y(a)+(t-a) y^{\prime}(a) \leqq y^{\prime}(a)[B+t-a] \leqq 2 t y^{\prime}(a) .
$$

and it follows that

$$
1 \leqq \frac{y(b)}{y(x)}+\left[2 y^{\prime}(a)\right]^{2 n} \int_{b}^{\infty} p(t) t^{2 n+1} d t+\frac{x y^{\prime}(x)}{y(x)},
$$

and thus

$$
1-\epsilon \leqq \frac{y(b)}{y(x)}+\frac{x y^{\prime}(x)}{y(x)} .
$$

If $y(x)$ is not bounded for $x \rightarrow \infty$, we therefore must have 


$$
1 \geqq \liminf _{x \rightarrow \infty} \frac{x y^{\prime}(x)}{y(x)} \geqq 1-\epsilon,
$$

where the left hand-inequality follows from the concavity of the curve $\zeta=y(x)$. This shows that unless $y(x)$ is bounded it must be such that

$$
\lim _{x \rightarrow \infty} \frac{x y^{\prime}(x)}{y(x)}=1 \text {. }
$$

To show that this is equivalent to (15), we choose $b$ large enough so that $x y^{\prime}(x)>(1-\epsilon) y(x)$ for $x \geqq b$. We then have

$$
(1-\epsilon)\left[y^{\prime}(b)-y^{\prime}(x)\right]=(1-\epsilon) \int_{b}^{x} p y^{2 n+1} d t \leqq \int_{b}^{x} t p y^{2 n} y^{\prime} d t \leqq y^{\prime}(b) \int_{b}^{x} t p y^{2 n} d t .
$$

Since, as shown above,

$$
\int_{b}^{x} t p y^{2 n} d t \leqq\left[2 y^{\prime}(a)\right]^{2 n} \int_{b}^{\infty} t^{2 n+1} p(x) d t<\epsilon,
$$

this leads to

$$
(1-2 \epsilon) y^{\prime}(b) \leqq(1-\epsilon) y^{\prime}(x) .
$$

Hence, $y^{\prime}(x)$ has a positive lower bound as $x \rightarrow \infty$. But $y^{\prime}(x)$ is monotonically decreasing as $x$ increases, and it follows that $y^{\prime}(x)$ tends to a positive limit as $x \rightarrow \infty$. Since $x y^{\prime}(x)[y(x)]^{-1} \rightarrow 1$ for $x \rightarrow \infty$, this completes the proof of Theorem IV.

It may be remarked that Theorems III and IV imply the following result.

If equation (1) has one solution with the asymptotic behavior (15), then all other nonoscillatory solutions either show the same behavior, or else are bounded.

6. We now turn to the problem of minimizing the Rayleigh quotient (3) within the family of functions which belong to class $D^{\prime}$ in $[a, b]$ and satisfy certain homogeneous boundary conditions. While, with very minor modifications, the treatment of the problem to be given here can be applied to the most general type of such boundary conditions, we restrict ourselves to the following two cases which are of special relevance to the oscillation problem of equation (1):

$$
\begin{array}{ll}
J(y)=\min , & y(a)=0 ; \\
J(y)=\min , & y(a)=y(b)=0 .
\end{array}
$$

We first show that, under these conditions, there exists a positive lower bound for $J(y)$. Since the side conditions in (20) are more stringent than in (19), it is sufficient to do this for problem (19). We denote by $\mu$ the lowest eigenvalue of the linear differential system

$$
\left(x^{-n} u^{\prime}\right)^{\prime}+\mu p(x) u(x)=0, \quad u(a)=u^{\prime}(b)=0,
$$


and we use the well-known fact [2] that $\mu>0$ and that, for any function $v(x)$ of $D^{\prime}[a, b]$ which vanishes at $x=a$, the inequality

$$
\mu \int_{a}^{b} p v^{2} d x \leqq \int_{a}^{b} x^{-n v^{\prime 2}} d x \quad(0<a<b<\infty)
$$

holds. If $y(x)$ vanishes at $x=a$ and belongs to $D^{\prime}[a, b]$, we may set $v(x)$ $=y^{n+1}(x)$. This yields

$$
\mu \int_{a}^{b} p y^{2 n+2} d x \leqq(n+1)^{2} \int_{a}^{b} x^{-n} y^{2 n} y^{\prime^{2}} d x
$$

Since

$$
y^{2}(x)=\int_{a}^{x} y^{\prime} d t^{2} \leqq(x-a) \int_{a}^{x} y^{\prime 2} d t<x \int_{a}^{x} y^{\prime 2} d t,
$$

it follows that

$$
\begin{aligned}
\mu \int_{a}^{b} p y^{2 n+2} d x & \leqq(n+1)^{2} \int_{a}^{b} y^{\prime 2}(x)\left(\int_{a}^{x} y^{\prime 2}(x) d t\right)^{n} d x \\
& =(n+1)\left(\int_{a}^{x}{y^{\prime 2}}^{2} d x\right)^{n+1} .
\end{aligned}
$$

Hence,

$$
(n+1) J(y) \geqq \mu,
$$

and the existence of a positive lower bound for $J(y)$ is established.

If we set $\lambda=\inf J(y)$, where $y(x)$ ranges over all functions subject to the admissibility conditions of problem (19), we have $\lambda>0$ and there will exist a sequence $\left\{y_{\nu}(x)\right\}$ such that $\lim _{\nu \rightarrow \infty} J\left(y_{\nu}\right)=\lambda$. A similar statement applies to problem (20); since the discussion of (19) and (20) is almost exactly alike, we shall refer to problem (20) only when there is a difference in the treatment of the two problems. The following lemma shows that the minimizing sequence $\left\{y_{\nu}(x)\right\}$ may be replaced by another sequence $\left\{u_{\nu}(x)\right\}$ of functions of $C^{\prime \prime}[a, b]$ which satisfy the boundary conditions $u_{\nu}(a)=u_{\nu}^{\prime}(b)=0$.

Lemma III. If $y(x) \in C^{\prime}[a, b]$ and

$$
\int_{a}^{b} y^{\prime 2} d x=1
$$

then

$$
J(u) \leqq \alpha \leqq J(y),
$$

where $u(x)$ and $\alpha$ are, respectively, the first eigenfunction and the first eigenvalue of the linear differential system 


$$
u^{\prime \prime}(x)+\alpha p(x) y^{2 n}(x) u(x)=0, \quad u(a)=u^{\prime}(b)=0 .
$$

By classical results, we have

$$
\alpha \int_{a}^{b} p y^{2 n} u^{2} d x=\int_{a}^{b} u^{\prime 2} d x, \quad \alpha \int_{a}^{b} p y^{2 n} v^{2} d x \leqq \int_{a}^{b}{v^{\prime 2}}^{2} d x,
$$

where $v(x)$ is any function of $D^{\prime}[a, b]$ which vanishes for $x=a$. If we set $y(x)$ $=v(x)$, we have, in particular,

$$
\alpha \int_{a}^{b} p y^{2 n+2} d x \leqq \int_{a}^{b} y^{\prime 2} d x=1,
$$

which is equivalent to the right-hand inequality (24). Using this, and the Hölder inequality, we obtain

$$
\begin{aligned}
\left(\int_{a}^{b}{u^{\prime 2}}^{2} d x\right)^{n+1}=\left(\alpha \int_{a}^{b} p y^{2 n} u^{2} d x\right)^{n+1} & \leqq \alpha\left(\alpha \int_{a}^{b} p y^{2 n+2} d x\right)^{n} \int_{a}^{b} p u^{2 n+2} d x \\
& \leqq \alpha \int_{a}^{b} p u^{2 n+2} d x,
\end{aligned}
$$

and this completes the proof of Lemma III.

In view of (24), the original minimizing sequence $\left\{y_{\nu}(x)\right\}^{\prime}$ for problem (19) - which may be assumed to be normalized by condition (23) - can be replaced by $\left\{u_{\nu}(x)\right\}$, where $u_{\nu}(x)$ is the solution of (25) for $y(x)=y_{\nu}(x)$. If $\alpha_{\nu}$ is the corresponding eigenvalue, we have

$$
\lim _{\nu \rightarrow \infty} J\left(y_{\nu}\right)=\lim _{\nu \rightarrow \infty} \alpha_{\nu}=\lim _{\nu \rightarrow \infty} J\left(u_{v}\right)=\lambda .
$$

The system (25) may be replaced by the equivalent integral equation

$$
u(t)=\alpha \int_{a}^{b} p(x) g(x, t) y^{2 n}(x) u(x) d x,
$$

where

$$
\begin{aligned}
& g(x, t)=x-a, \\
& a \leqq x \leqq t, \\
& g(x, t)=t-a, \\
& t \leqq x \leqq b .
\end{aligned}
$$

In the case of the boundary conditions $u(a)=u(b)=0$ i.e., problem (20), the corresponding function $g_{1}(x, t)$ is given by

$$
\begin{aligned}
& (b-a) g_{1}(x, t)=(x-a)(b-t), \\
& a \leqq x \leqq t, \\
& (b-a) g_{1}(x, t)=(t-a)(b-x), \\
& t \leqq x \leqq b .
\end{aligned}
$$

It is easily confirmed that

$$
\left|g\left(x, t_{2}\right)-g\left(x, t_{1}\right)\right| \leqq\left|t_{2}-t_{1}\right|
$$


and

$$
\left|g_{1}\left(x, t_{2}\right)-g_{1}\left(x, t_{1}\right)\right| \leqq\left|t_{2}-t_{1}\right|,
$$

if $t=t_{1}$ and $t=t_{2}$ are two arbitrary points in $[a, b]$. In both cases, we may therefore conclude that

$$
\left|u\left(t_{2}\right)-u\left(t_{1}\right)\right| \leqq \alpha\left|t_{2}-t_{1}\right| \int_{a}^{b} p y^{2 n} u d x,
$$

whence

$$
\begin{aligned}
\left|u\left(t_{2}\right)-u\left(t_{1}\right)\right|^{n+1} & \leqq \alpha^{n+1}\left|t_{2}-t_{1}\right|^{n+1}\left(\int_{a}^{b} p y^{2 n+2} d x\right)^{n} \int_{a}^{b} p u^{n+1} d x \\
& \leqq \alpha^{n+1}\left|t_{2}-t_{1}\right|^{n+1}\left(\int_{a}^{b} p y^{2 n+2} d x\right)^{n}\left(\int_{a}^{b} p d x\right)^{1 / 2}\left(\int_{a}^{b} p u^{2 n+2} d x\right)^{1 / 2} .
\end{aligned}
$$

In view of (26), we thus have

$$
\left|u\left(t_{2}\right)-u\left(t_{1}\right)\right|^{n+1} \leqq \alpha\left|t_{2}-t_{1}\right|^{n+1}\left(\int_{a}^{b} p d x \int_{a}^{b} p u^{2 n+2} d x\right)^{1 / 2} .
$$

If $u(x)$ is also normalized by (23)-or, what amounts to the same thing, by the condition

$$
\alpha \int_{a}^{b} p y^{2 n} u^{2} d x=1
$$

this reduces to

$$
\left|u\left(t_{2}\right)-u\left(t_{1}\right)\right| \leqq\left[\alpha \int_{a}^{b} p d x\right]^{1 /(n+1)}\left|t_{2}-t_{1}\right| .
$$

For each function $u_{\nu}(x)$ of our minimizing sequence we now set up a sequence of functions $v_{\nu \mu}(x)$ defined by

$$
v_{\nu 1}(x)=u_{\nu}(x), \quad v_{\nu \mu}^{\prime \prime}+\alpha_{\nu \mu} p_{\nu, \mu-1}^{2 n} v_{\nu \mu}=0, \quad v_{\nu \mu}(a)=v_{\nu \mu}^{\prime}(b), \quad \int_{a}^{b} v_{\nu \mu}^{\prime 2} d x=1,
$$

where $\alpha_{\nu \mu}$ is again the lowest eigenvalue of the system. By Lemma III, $\alpha_{\nu, \mu} \leqq \alpha_{\nu, \mu-1}$ and $\alpha_{\nu 1} \leqq \alpha_{\nu}$. Since $\lambda$ is a lower bound for all $\alpha_{\nu \mu}$, it thus follows from (27) that the diagonal sequence $\alpha_{\nu \nu}$ has the limit $\lambda$. Applying (30) to the functions $v_{\nu}(x) \equiv v_{\nu \nu}(x)$ and observing that the $\alpha_{\nu \nu}$ are uniformly bounded, we obtain

$$
\left|v_{\nu}\left(t_{2}\right)-v_{\nu}\left(t_{1}\right)\right| \leqq M\left|t_{2}-t_{1}\right|,
$$

where $M$ is independent of $t_{1}, t_{2}$, and $\nu$. 
The sequence $\left\{v_{\nu}(x)\right\}$ is thus found to be equicontinuous in $[a, b]$. Because of

$$
v_{v}^{2}(x) \leqq(x-a) \int_{a}^{x} v_{\nu}^{\prime 2} d x \leqq b-a
$$

the functions $v_{\nu}(x)$ are moreover uniformly bounded in this interval. By classical results there will therefore exist a subsequence $\left\{v_{\nu^{\prime}}(x)\right\}$ which converges uniformly to a continuous function $Y(x)$. By (29), we have

$$
\alpha_{v v} \int_{a}^{b} p u_{v, \nu-1}^{2 n} v_{\nu}^{2} d x=1
$$

Taking, if necessary, a sub-subsequence for which $u_{\nu, \nu-1}$ converges---say to a function $Y_{1}(x)$-we obtain

$$
\lambda \int_{a}^{b} p Y_{1}^{2 n} Y^{2} d x=1 .
$$

This shows that neither of the continuous functions $Y(x), Y_{1}(x)$ can be identically zero. Since the solutions of (25) are positive (if $\left.u^{\prime}(a)>0\right)$ and concave in $(a, b)$ it follows, furthermore, that $Y(x)$ and $Y_{1}(x)$ have the same properties. We also remark that it follows from the uniform convergence in the closed interval $[a, b]$ that both functions satisfy the boundary conditions (25).

(31) and the last remark show that $Y(x)$ and $Y_{1}(x)$ must be identical. By (31), we have

$$
\lambda^{-n-1}=\left(\int_{a}^{b} p Y_{1}^{2 n} Y^{2} d x\right)^{n+1} \leqq\left(\int_{a}^{b} p Y_{1}^{2 n+2} d x\right)^{n} \int_{a}^{b} p Y^{2 n+2} d x .
$$

Since, in view of the normalization of $Y$ and $Y_{1}$,

$$
J(Y)=\frac{\left(\int_{a}^{b} Y^{\prime 2} d x\right)^{n+1}}{\int_{a}^{b} p Y^{2 n+2} d x}=\frac{1}{\int_{a}^{b} p Y^{2 n+2} d x},
$$

and similarly for $Y_{1}$, it follows that

$$
\lambda^{-n-1} \leqq J^{-n}(Y) J^{-1}\left(Y_{1}\right) \leqq \lambda^{-n-1},
$$

where the last inequality is due to the fact that both $Y$ and $Y_{1}$ are admissible functions in problem (19). This shows that the Hölder inequality used in (32) must reduce to an equality and that, consequently $Y(x)$ and $Y_{1}(x)$ must be proportional. But both are positive and both are normalized by (23); hence, they are identical. 
If we pass to the limit $\nu \rightarrow \infty$ in (28), we find that $Y(x)$ satisfies the integral equation

$$
Y(t)=\lambda \int_{a}^{b} p(x) g(x, t) Y^{2 n+1}(x) d x .
$$

This shows that $Y(x)$ has two continuous derivatives and that it is a solution of the corresponding differential equation

$$
Y^{\prime \prime}+\lambda p Y^{2 n+1}=0 .
$$

Accordingly, the function $y(x)=\lambda^{1 / 2 n} Y(x)$ will be a solution of

$$
y^{\prime \prime}+p y^{2 n+1}=0
$$

i.e., of equation (1).

The following theorem summarizes our results.

THEOREM V. For each pair of numbers $a, b$ such that $0<a<b<\infty$, equation (1) has two solutions, $y(x)$ and $u(x)$, which are positive in $(a, b)$ and satisfy the conditions

$$
y(a)=y^{\prime}(b)=0, \quad u(a)=u(b)=0 .
$$

If $J(y)$ denotes the Rayleigh quotient (3), these functions are characterized by the minimum properties

$$
J(y) \leqq J(\sigma), \quad J(u) \leqq J(\tau),
$$

where, except for the conditions $\sigma(a)=\tau(a)=\tau(b)=0, \sigma(x)$ and $\tau(x)$ are arbitrary functions of class $D^{\prime}[a, b]$.

7. In a previous paper [4] it was shown that in the case of a linear equation (1)-i.e., for $n=0$ - the minimal value of $J(y)$ plays a central role in the oscillation problem of the equation. It was found that the equation is nonoscillatory in $(a, \infty)$-that is, no solution has more than one zero in this interval if, and only if, $J(y) \geqq 1$ for all $b$ such that $b>a$. In the case of a positive $n$, the connection between the oscillation problem of (1) and the extremal problem involving $J(y)$ cannot be expected to be quite so simple, the main reason being that as shown by Theorem $\mathrm{V}$-there does not exist an interval $(a, \infty)$ in which no solution of (1) has more than one zero. In fact, as will be shown in $\$ 8$, there does not exist an interval $(a, \infty)$ in which no solution of (1) has more than $m$ zeros, where $m$ is an arbitrary fixed positive integer.

Another major difficulty in establishing the precise relationship between the extremal problem for $J(y)$ and the oscillation problem of (1) is the fact, demonstrated by the example discussed in $\$ 2$, that the solution of (1) determined by $y(a)=y(b)=0, y(x)>0(a<x<b)$ is not necessarily unique. For our purposes it would be sufficient if this uniqueness property could be established at least for sufficiently large values of $b$, and only for the boundary conditions 
$y(a)=y^{\prime}(b)=0, y(x)>0(a \leqq x<b)$ (which is a less stringent requirement). The importance of this uniqueness property is indicated by the following results.

THEOREM VI. If equation (1) has a solution $y(x)$ such that $y(a)=0, y(x)>0$ for $x>a$ and if, for sufficiently large $b$, there exists not more than one solution $y_{b}(x)$ of (1) such that $y_{b}(a)=y_{b}^{\prime}(b)=0$ and $y_{b}(x)>0$ in $(a, b)$, then there exists $a$ constant $\alpha(\alpha>0)$ such that

$$
\alpha \int_{a}^{b} p \sigma^{2 n+2} d x \leqq\left(\int_{a}^{b}{\sigma^{\prime 2}}^{2} x\right)^{n+1}
$$

for any $b>a$ and for any function $\sigma(x)$ of $D^{\prime}[a, b]$ for which the right-hand integral exists.

By choosing suitable functions $\sigma(x)$, it is possible to obtain a considerable amount of information about the function $p(x)$. For instance, if we set $\sigma(x)=(x-a)(t-a)^{-1}$ in $[a, t]$ and $\sigma(x) \equiv 1$ in $[t, b]$, where $a<t<b$, and extend the integral on the left-hand side of $(34)$ only over $[t, b]$, we obtain

$$
\alpha \int_{t}^{b} p(x) d x \leqq(t-a)^{-n-1} .
$$

Since $b$ may be taken arbitrarily large, this yields the inequality

$$
(t-a)^{n+1} \int_{t}^{\infty} p(x) d x \leqq \alpha^{-1}<\infty,
$$

which is thus established for any $p(x)$ satisfying the hypotheses of Theorem VI. If, for $t \leqq x \leqq b$, the definition of $\sigma(x)$ is changed to $(x-a)^{\nu}(t-a)^{-\nu}$ with suitable values of $\nu$, the same procedure leads to

$$
\int_{t}^{\infty} x^{n+1-e} p(x) d x<\infty
$$

for any positive $\epsilon$.

To prove Theorem VI we note that, by the existence theorem for equation (1), the first zero in $(a, \infty)$ of the derivative of the solution of (1) given by $y(a)=0, y^{\prime}(a)=A$ moves continuously as $A$ varies continuously from 0 through positive values to $\infty$. An elementary consideration shows that $A \rightarrow \infty$ corresponds to $b \rightarrow a$, if $x=b$ denotes the zero in question. If $b \rightarrow \infty$ for $A \rightarrow 0$, it would follow that the derivatives of all solutions of (1) such that $y(a)=0, y^{\prime}(a)>0$ have at least one zero in $(a, \infty)$, since for sufficiently large $b$ the quantity $A$ is assumed to be a single-valued function of $b$. But this would contradict the assumption that there exists a solution which vanishes at $x=a$ and is positive for $x>a$ (such a solution necessarily has a positive derivative in $(a, \infty)$ ). It follows that $\lim A$ for $b \rightarrow \infty$ exists (for sufficiently large $b, A$ is a monotonic function of $b$ ) and is positive. 
The value of the functional $J(y)$ in Theorem $\mathrm{V}$ is decreasing for increasing $b$. Indeed, if the right-hand endpoint of the interval to which $J(y)$ refers is indicated by a subscript we have, by Theorem $\mathrm{V}$,

$$
J_{c}\left(y_{c}\right) \leqq J_{c}(\sigma) \text {, }
$$

where $\sigma(x)$ is any function satisfying the admissibility conditions. If $c>b$, we may set $\sigma(x)=y_{b}(x)$ in $a \leqq x \leqq b$, and $\sigma(x) \equiv y_{b}(b)$ in $b \leqq x \leqq c$. Since, clearly, $J_{c}(\sigma)<J_{b}\left(y_{b}\right)$, it follows from (37) that $J_{c}\left(y_{c}\right)<J_{b}\left(y_{b}\right)$.

Multiplying equation (1)-for $y=y_{b}$-with $y_{b}$ and integrating from $a$ to $b$, we obtain

$$
\int_{a}^{b} y_{b}^{\prime^{2}} d x=\int_{a}^{b} p y^{2 n+2} d x
$$

and (3) reduces therefore to

$$
J_{b}\left(y_{b}\right)=\left(\int_{a}^{b} y_{b}^{\prime 2} d x\right)^{n}
$$

Using the monontonicity of $J_{b}\left(y_{b}\right)$, we thus have

$$
\left(\int_{a}^{b} y_{c}^{\prime^{\prime 2}} d x\right)^{n}<\left(\int_{a}^{b} y_{c}^{\prime 2} d x\right)^{n}=J_{c}\left(y_{c}\right)<J_{b}\left(y_{b}\right), \quad a<b<c .
$$

As shown above $A_{0}=\lim _{c \rightarrow \infty} y_{c}^{\prime}(a)$ exists and is positive. In every finite interval, $y_{c}(x)$ will therefore converge uniformly to the solution $y_{0}(x)$ of $(1)$ for which $y_{0}(a)=0, y_{0}^{\prime}(a)=A_{0}$; evidently, $y_{0}(x)>0$ in $(a, \infty)$. By (38), we have

$$
\int_{0}^{b} y_{0}^{\prime 2} d x<J_{b}\left(y_{b}\right) \text {. }
$$

If we now let $b \rightarrow \infty$, the right-hand side of this inequality decreases while the left-hand side increases. This shows that $\alpha=\lim _{b \rightarrow \infty} J_{b}\left(y_{b}\right)$ is positive (and, incidentally, that $\int_{a}^{\infty} y_{0}^{\prime 2} d x$ exists). In view of the minimum property of $J_{b}\left(y_{b}\right)$ set forth in Theorem V, this proves Theorem VI.

It may be noted that the proof of Theorem VI does not make full use of the uniqueness of the solutions $y_{b}$ for large $b$. All that was used was the fact -implied by the uniqueness-that $y_{b}^{\prime}(a)$ does not tend to zero for $b \rightarrow \infty$. The conclusion of Theorem VI will therefore remain valid under this weaker assumption. The following statement refers to a case in which the validity of this assumption can be directly verified.

If (1) has two solutions $u(x), v(x)$ such that $u(a)=v(a)=0$ and $0<v(x)<u(x)$ in $(a, \infty)$, then the conclusion of Theorem VI holds.

Indeed, suppose that $0<y_{b}^{\prime}(a)<v^{\prime}(a)$ for any $b>a$. By Lemma II, the curves $\zeta=y_{b}(x)$ and $\zeta=v(x)$ cannot intersect in $(a, b)$, and we must therefore have $y_{b}(b)<v(b)$. For $x>b, y_{b}(x)$ decreases and, because of the concavity of the curve $\zeta=y_{b}(x)$, this curve will intersect the $x$-axis at a point $x=c(c>b)$. In $(b, c), v(x)$ is increasing and the entire arch of $\zeta=y_{b}(x)$ in $(a, c)$ will thus lie 
below the curve $\zeta=v(x)$. But this again violates Lemma II, and the assumption $0<y_{b}^{\prime}(a)<v^{\prime}(b)$ is thus shown to lead to an absurdity. Hence, $y_{b}^{\prime}(a)$ $>v^{\prime}(a)>0$ for all $b>a$, and the statement is proved.

It may be remarked that the existence of two solutions $u(x), v(x)$ with the indicated properties has thus been found to imply condition (36). This may be compared with the fact, proved in $\$ 5$, that the existence of two such solutions is guaranteed by condition (14).

8. The following result shows that the "nonoscillatory" character of a nonlinear equation of the form (1) is something very different from the corresponding property of a linear equation.

THEOREM VII. For any $a, b$ such that $0<a<b<\infty$ and for any non-negative integer $m$, there exists a solution of (1) which vanishes at $x=a$ and $x=b$, and has precisely $m$ zeros in $(a, b)$.

Since the case $m=0$ is contained in Theorem $V$, we may assume that $m>0$. The proof will be based on an extremal problem which generalizes the extremal problem in Theorem $\mathrm{V}$, and whose solution will exhibit the required properties. We choose $m$ distinct points $x=a$ in $(a, b)$ such that $a_{0}=a<a_{1}$ $<a_{2}<\cdots<a_{m}<b=a_{m+1}$, and we denote by $J_{\nu}(\sigma)$ the Rayleigh quotient (3) for the interval $\left[a_{\nu-1}, a_{\nu}\right]$, where $\sigma \in D^{\prime}[a, b]$ and $\sigma\left(a_{\nu}\right)=0$ for $\nu=0,1, \cdots$, $m+1$. The appropriate extremal problem is

$$
\sum_{v=1}^{m+1}\left[J_{\nu}(\sigma)\right]^{1 / n}=\min ,
$$

where $\sigma$ ranges over all functions with the indicated properties, and the $a_{\nu}(\nu=1, \cdots, m)$ may be chosen in any way compatible with the above assumptions.

Theorem $\mathrm{V}$ shows that, for a fixed choice of $a_{1}, \cdots, a_{m}$, the minimum of (39) will be attained by $m+1$ solutions of (1), each vanishing at two neighboring points and not vanishing in between. Our task thus reduces to the problem of minimizing the function of the $m$ variables $a_{1}, \cdots, a_{m}$ defined in this way. Since elementary considerations show that $\min J(y)$ tends to $\infty$ if the length of the interval of integration shrinks to zero, it is clear that the limit of a converging sequence of sets $\left(a_{1}, \cdots, a_{m}\right)$ for which (39) tends to its greatest lower bound, will consist of $m$ distinct points. If we can show that, at each point $a_{\nu}$, the functions $y_{\nu}(x)$ obtained in this way are subject to the conditions $y_{\nu}^{\prime 2}\left(a_{v}\right)=y_{\nu+1}^{\prime 2}\left(a_{v}\right)$, the existence of a solution of (1) with the properties indicated in Theorem VII will follow; clearly, if we let positive and negative arches $\zeta= \pm y(x)$ alternate they will form a regular solution of $(1)$ in $[a, b]$.

In order to derive the required differentiation formulas of $J(y)$ with respect to the limits of the interval of integration, we denote by $y(x)$ and $y_{1}(x)$ two solutions of (1) such that $y(\alpha)=y_{1}(\alpha)=y(\beta)=y_{1}(\beta+\epsilon)=0$ $(0<\alpha<\beta<\beta+\epsilon<\infty)$, where $y(x)$ and $y_{1}(x)$ are positive in $(\alpha, \beta)$ and $(\alpha, \beta+\epsilon)$, respectively. By Theorem $\mathrm{V}$ these solutions exist for any $\alpha, \beta$, subject to the 
above conditions, and the existence theorem for equation (1) shows that $y_{1}(\beta+\epsilon)-$ or, if $y_{1}(\beta+\epsilon)$ is not uniquely determined by the conditions imposed on it, one of the possible solutions $y_{1}(\beta+\epsilon)$-is a differentiable function of $\epsilon$. If we write $y_{\epsilon}(x) \equiv \lim _{\epsilon \rightarrow 0} \epsilon^{-1}\left[y_{1}(x)-y(x)\right]$ and note that

$$
\int_{\alpha}^{\beta+\epsilon} p y_{1}^{2 n+1} d x-\int_{\alpha}^{\beta} p y^{2 n+1} d x=\int_{\alpha}^{\beta+\epsilon} y_{1}^{\prime^{2}} d x-\int_{\alpha}^{\beta} y^{\prime^{2}} d x
$$

an elementary computation shows that

$$
2 \int_{\alpha}^{\beta} y^{\prime} y_{\epsilon}^{\prime} d x+y^{\prime 2}(\beta)=(2 n+1) \int_{\alpha}^{\beta} p y^{2 n+1} y_{e} d x .
$$

Since, in view of (1), $y_{e}$ must be a solution of

$$
y_{e}^{\prime \prime}+(2 n+1) p y^{2 n} y_{e}=0,
$$

we have

$$
\int_{\alpha}^{\beta} y^{\prime} y_{\epsilon}^{\prime} d x=(2 n+1) \int_{\alpha}^{\beta} p y^{2 n+1} y_{\epsilon} d x
$$

and thus

$$
2 n \int_{\alpha}^{\beta} p y^{2 n+1} y_{\epsilon} d x=-{y^{\prime 2}}^{2}(\beta) .
$$

Another elementary computation shows that

$$
\begin{aligned}
\left.\frac{\partial}{\partial \epsilon} \int_{\alpha}^{\beta+\epsilon} y_{1}^{\prime 2} d x\right|_{\epsilon=0} & =2 \int_{\alpha}^{\beta} y^{\prime} y^{\prime} d x+y^{\prime^{2}}(\beta) \\
& =2(2 n+1) \int_{\alpha}^{\beta} p y^{2 n+1} y_{\epsilon} d x+y^{\prime}(\beta) \\
& =-\frac{n+1}{n}{y^{\prime 2}}^{2}(\beta)
\end{aligned}
$$

and

$$
\left.\frac{\partial}{\partial \epsilon} \int_{\alpha}^{\beta+\epsilon} p y_{1}^{2 n+2} d x\right|_{\epsilon=0}=2(n+1) \int_{\alpha}^{\beta} p y^{2 n+1} y_{e} d x=-\frac{n+1}{n} y^{\prime 2}(\beta) .
$$

Combining these identities and observing the definition $(\beta)$ of $J(y)$, we obtain

$$
\left.\frac{\partial}{\partial \epsilon}\left\{[J(y)]^{1 / n}\right\}\right|_{\epsilon=0}=-(n+1){y^{\prime 2}}^{2}(\beta) \text {. }
$$

If $\alpha$ is made to vary while $\beta$ is kept fixed, the same argument shows that the corresponding derivative is $(n+1) y^{\prime^{2}}(\alpha)$. Hence, if $a_{\nu}$ is replaced by $a_{\nu}+\epsilon$ while all other points $a_{\mu}(\mu \neq \nu)$ are kept fixed, the variation of the expression 
(39) is $\epsilon(n+1)\left[y_{\nu+1}^{\prime 2}\left(a_{v}\right)-y_{\nu}^{\prime 2}\left(a_{v}\right)\right]+o(\epsilon)$, and it follows that for the minimal set $\left\{a_{\mu}\right\}$ we must have $y_{\nu+1}^{\prime 2}\left(a_{\nu}\right)=y_{\nu}^{\prime 2}\left(a_{\nu}\right)$. This completes the proof of Theorem VII.

9. As mentioned in $\S 7$, nonoscillation of the solutions of a linear equation (1) (i.e. for $n=0)$ in $(\alpha, \infty)$ is equivalent to the inequality $J(y) \geqq 1$ for all $b>a$, where $J(y)$ is the functional defined in Theorem $\mathrm{V}$. While, for reasons indicated in $\$ 7$, no such simple connection between nonoscillation and the lower bound for $J(y)$ can be expected in the general case, Theorem VI shows that, under certain additional conditions, the existence of a positive constant $A$ such that $J(y) \geqq A$ for all $b>a$ is a necessary condition for the existence of a nonoscillatory solution of (1) which vanishes at $x=a$. There are indications that the inequality $J(y)>A>0$ may be valid under the sole assumption that (1) have a nonoscillatory solution of this type. On the other hand, as a detailed discussion of equation (7) shows, the mere existence of such a constant $A$ is not a sufficient condition for the presence of a solution of this kind. The consideration of a number of examples makes it seem likely that there exist two universal constants $A_{1}$ and $A_{2}\left(0<A_{1}<A_{2}<\infty\right)$ such that $J(y) \geqq A_{1}$ is a necessary, and $J(y) \geqq A_{2}$ a sufficient condition, but we are unable to prove this.

In $\$ 7$ it was shown (formula (35)) that the existence of a positive constant $A$ with the above properties implies

$$
\limsup _{x \rightarrow \infty} x^{n+1} \int_{x}^{\infty} p(t) d t<\infty .
$$

The following theorem shows that there will be such a constant if

$$
\int^{\infty} x^{n+1} p(x) d x<\infty .
$$

Theorem VIII. If $J(y)$ is the minimum value defined in Theorem $\mathrm{V}$ and $A=\lim _{b \rightarrow \infty} J(y)$ for fixed $a$, then

$$
\begin{aligned}
& A(x-a)^{n+1} \int_{x}^{\infty} p(t) d t \leqq 1, \\
& A \int_{a}^{\infty}(x-a)^{n+1} p(x) d x \geqq 1 .
\end{aligned}
$$

To prove (41), we go back to the relation (22) between $J(y)$ and the lowest eigenvalue of the differential system (21). By the substitution $t=(x-c)^{n+1}$ $(0<c<a)$, equation $(21)$ is transformed into

$$
\ddot{u}+(n+1)^{-2}(x-c)^{-n} p u=0,
$$

where the dot indicates differentiation with respect to $t$, and the boundary conditions become $u\left(t_{1}\right)=u\left(t_{2}\right)=0$, with $t_{1}=(a-c)^{n+1}, t_{2}=(b-c)^{n+1}$. It is well 
known (cf., e.g., [5]) and easily proved, that the existence of such a solution implies the condition

$$
\mu(n+1)^{-2} \int_{t_{1}}^{t_{2}}\left(t-t_{1}\right)(x-c)^{-n} p d t \geqq 1 .
$$

If we return to the variable $x$ and utilize (22), we obtain

$$
J(y) \int_{a}^{b}\left[(x-c)^{n+1}-(a-c)^{n+1}\right] p(x) d x \geqq 1 .
$$

Letting $c \rightarrow a$, we arrive at (41).

We close this section with a result which shows that the expression appearing in (40) is related to the rate of growth of the solutions of (1).

Theorem IX. If

$$
\liminf _{x \rightarrow \infty} x^{n+1} \int_{x}^{\infty} p(t) d t>0,
$$

and $y(x)$ is a nonoscillatory solution of (1), then

$$
y(x) \leqq c x^{1 / 2},
$$

where $c$ is a positive constant.

We assume that $y(x)>0$ for $x \geqq a$, and we write (1) in the form

$$
y(t)=y(a)+\int_{a}^{t}(x-a) p y^{2 n+1} d x+(t-a) \int_{t}^{b} p y^{2 n+1} d x+(t-a) y^{\prime}(b) .
$$

Since $y(x)$ increases with $x$, we have

$$
1 \geqq y^{2 n}(t)(t-a) \int_{t}^{b} p(x) d x
$$

for arbitrarily large $b$; hence,

$$
1 \geqq\left[\frac{y^{2}(t)}{t-a}\right]^{n}(t-a)^{n+1} \int_{t}^{\infty} p(x) d x .
$$

If this is compared with (42), the result follows.

10. In those cases in which the general solution of an equation (1) can be found, Theorem $\mathrm{V}$ becomes a source of sharp integral inequalities of the type

$$
\int_{a}^{b} p(x) y^{2 n}(x) d x \leqq c\left(\int_{a}^{b} y^{\prime 2}(x) d x\right)^{n} .
$$

We consider here a few examples of this kind. It may be noted that in these cases the solutions which are positive in $(a, b)$ and satisfy either set of boundary conditions (33) are unique. 
If $y(x)$ is a solution of

$$
y^{\prime \prime}+n y^{2 n-1}=0,
$$

$n \geqq 1$,

which satisfies $y(a)=y^{\prime}(b)=0$, we have

$$
\int_{a}^{b} y^{\prime 2} d x=n \int_{a}^{b} y^{2 n} d x
$$

and

$$
y^{\prime 2}(x)+y^{2 n}(x)=y^{\prime 2}(a)=y^{2 n}(b) .
$$

Hence,

$$
\int_{a}^{b} y^{\prime^{2}} d x+\int_{a}^{b} y^{2 n} d x=(b-a){y^{\prime 2}}^{2}(a)
$$

and, if this is combined with (44),

$$
(n+1) \int_{a}^{b} y^{\prime 2} d x=n(b-a) y^{\prime \prime}(a) .
$$

Thus,

$$
J(y)=n^{n}(n+1)^{1-n}(b-a)^{n-1}\left[y^{\prime}(a)\right]^{2 n-2} .
$$

By (45), we have

$$
b-a=\int_{0}^{y(b)} \frac{d y}{\left[y^{2 n}(b)-y^{2 n}\right]^{1 / 2}}=[y(b)]^{1-n} \int_{0}^{1} \frac{d t}{\left(1-t^{2 n}\right)^{1 / 2}}
$$

and this leads to the following result.

If $y(a)=0$ and $y(x) \in D^{\prime}[a, b]$, then

$$
\int_{a}^{b} y^{2 n} d x \leqq k\left(\int_{a}^{b} y^{\prime 2} d x\right)^{n}
$$

where

$$
k=(n+1)^{n-1} n^{-n}(b-a)^{n+1} \gamma^{-2 n},
$$

and

$$
\gamma=\int_{0}^{1} \frac{d t}{\left(1-t^{2 n}\right)^{1 / 2}}
$$

(46) will become an equality if, and only if, $y(x)$ is a constant multiple of the solution of $y^{\prime \prime}+n y^{2 n-1}=0$ which is positive in $(a, b)$ and is subject to the conditions $y(a)=y^{\prime}(b)=0$.

The next example concerns the equation

$$
w^{\prime \prime}+n x^{-2 n-2} w^{2 n-1}=0,
$$

whose general solution is of the form 


$$
w(x)=x y\left(\frac{1}{a}-\frac{1}{x}\right)
$$

where $y(x)$ is the general solution of (43). We consider here only the boundary conditions $w(a)=w(b)=0$, since the determination of the exact constant in the case $w(a)=w^{\prime}(b)=0$ involves the solution of a transcendental equation. If $t=a^{-1}-x^{-1}$, we have

$$
\int_{a}^{b} w^{\prime 2} d x=\int_{a}^{c}(\dot{y}+x y)^{2} d t=\int_{a}^{c} \dot{y}^{2} d t+\left[x y^{2}\right]_{a}^{c}=\int_{a}^{c} \dot{y}^{2} d t
$$

if $t=0$ and $t=c=a^{-1}-b^{-1}$ are two adjacent zeros of the corresponding solution $y(t)$ of (43). Since, moreover,

$$
\int_{a}^{b} x^{-2 n-2} w^{2 n} d x=\int_{a}^{c} y^{2 n} d t
$$

our inequality reduces to the analogue of (46) for the case $y(a)=y(b)=0$. Simple symmetry considerations show that in this case the constant $k$ appearing in (46) must be replaced by $2^{1-n} k$, and we arrive at the following result.

If $y(a)=y(b)=0$ and $y(x) \in D^{\prime}[a, b]$, then

$$
\int_{a}^{b} x^{-2 n-2} y^{2 n} d x \leqq k_{1}\left(\int_{a}^{b} y^{\prime 2} d x\right)^{n}
$$

where

$$
k_{1}=(n+1)^{n-1} n^{-n}\left(\frac{1}{a}-\frac{1}{b}\right)^{n+1} \gamma^{-2 n},
$$

and $\gamma$ is defined in (47). The sign of equality will hold only if $y(x)$ is the solution of (48) which is positive in $(a, b)$ and satisfies the required boundary conditions.

It may be pointed out that in accordance with Theorem VIII, the constant $k_{1}$ has a finite limit for $b \rightarrow \infty$, while the constant $k$ of (46) becomes infinitely large.

\section{BiBLIOGRAPHY}

1. F. V. Atkinson, On second-order non-linear oscillations, Pacific J. Math. vol. 5 (1955) pp. 643-647.

2. R. Courant and D. Hilbert, Methods of mathematical physics, New York, Interscience, 1953.

3. E. Hille, Non-oscillation theorems, Trans. Amer. Math. Soc. vol. 64 (1948) pp. 234-252.

4. Z. Nehari, Oscillation criteria for second-order linear differential equations, Trans. Amer. Math. Soc. vol. 85 (1957) pp. 428-445.

5. A. Wintner, On the Laplace-Fourier transcendents occurring in mathematical physics, Amer. J. Math. vol. 69 (1947) pp. 87-98.

Carnegie Institute of Technology, Pittsburgh, PA. 